$11-2015$

\title{
An Upscaled Approach for Transport in Media with Extended Tailing Due to Back-Diffusion Using Analytical and Numerical Solutions of the Advection Dispersion Equation
}

Jack C. Parker

University of Tennessee, Knoxville, jparker@utk.edu

Ungtae Kim

Cleveland State University, u.kim@csuohio.edu

Follow this and additional works at: https://engagedscholarship.csuohio.edu/encee_facpub

Part of the Environmental Engineering Commons

How does access to this work benefit you? Let us know!

Original Citation

Parker, J. C., and Kim, U. (2015). "An upscaled approach for transport in media with extended tailing due to back-diffusion using analytical and numerical solutions of the advection dispersion equation."

J.Contam.Hydrol., 182 157-172.

This Article is brought to you for free and open access by the Civil and Environmental Engineering at EngagedScholarship@CSU. It has been accepted for inclusion in Civil and Environmental Engineering Faculty Publications by an authorized administrator of EngagedScholarship@CSU. For more information, please contact library.es@csuohio.edu. 


\section{An upscaled approach for transport in media with extended tailing due to back-diffusion using analytical and numerical solutions of the advection dispersion equation}

Jack C. Parker, Ungtae Kim

\section{Introduction}

The most commonly used mathematical models for subsurface chemical transport are based on the mono-continuum advection dispersion equation ( $\mathrm{MADE}$ ), which assumes that chemical fluxes can be treated as the sum of advective and dispersive terms. Dispersive fluxes are approximated by analogy to Fick's law of diffusion as the product of a concentration gradient vector and a dispersion tensor, which attempts to describe the combined effects of molecular diffusion and hydrodynamic dispersion. With a uniform and constant dispersion coefficient in an infinite domain, Fick's law produces symmetrical concentration distributions in time and space with spreading rates proportional to the square root of 
time. However, highly heterogeneous systems often exhibit markedly asymmetric travel time distributions characterized by early breakthrough and extended tailing. That is, initial breakthrough at a distance $x$ downstream of a pulse injection location is often observed to occur at a fraction of $x / v^{\prime}$ (where $v^{\prime}$ is the retarded plume velocity) and contamination continues to be observed at times that are many multiples of $x / v^{\prime}$. Such observations are sometimes referred to as "anomalous" or "non-Fickian." The latter term has also been used to describe a variety of methods such as fractional-order ADEs, to more accurately represent complex field-scale behavior (e.g., Levy and Berkowitz, 2003; Bromly and Hinz, 2004; Berkowitz et al., 2006; Neuman and Tartakovsky, 2009; Dentz et al., 2011; Rubin et al., 2012). Therefore, we will avoid the term "non-Fickian" to prevent confusion between empirical observations and mathematical methods.

Nonequilibrium processes, such as diffusion between areas of high and low permeability, can also produce asymmetric travel time distributions and extended tailing associated with long-term back-diffusion of contaminants from low permeability zones, which can substantially impede aquifer restoration efforts (Ball et al., 1997; Liu and Ball, 2002; Chapman and Parker, 2005; Parker et al., 2008; Seyedabbasi et al., 2012). A number of bi-continuum models have been developed that consist of overlapping "mobile" and "immobile" continua coupled by diffusion or mass transfer equations (e.g., Coats and Smith, 1964; De Smedt and Wierenga, 1979; Rasmuson and Neretnieks, 1980; Tang et al., 1981; Gillham et al., 1984; Barker, 1985; Starr et al., 1985; Sudicky et al., 1985; Liu et al., 1998). Upscaled dispersion coefficients have been shown by a number of authors to predict the behavior of bi-continuum systems that are "equivalent" in some sense (e.g., same low-order moments) when employed in the mADE (e.g., Passioura, 1971; Bolt, 1979; Parker, 1984; Parker and Valocchi, 1986; van Genuchten and Dalton, 1986). Such studies imply that the MADE should be able to describe the behavior of systems that exhibit mass transfer limitations to a certain degree of approximation. Nevertheless, it is widely perceived that the mADE is categorically inapplicable to mobile-immobile type systems.

It is our objective in this paper to evaluate the extent to which the mADE can reasonably approximate transport in media characterized by diffusion-limited mass transfer and to identify model formulation and execution details that affect such performance for both analytical and numerical solution methods.

\section{Methodology}

\subsection{Formulation of transport equations for resident and flux concentrations}

The importance of distinguishing between resident and flux concentrations when dealing with heterogeneous aquifers is well established in the literature (Kreft and Zuber, 1978; Parker, 1984; Parker and van Genuchten, 1984; van Genuchten and Parker, 1984; Batu and van Genuchten, 1990; Roth and Jury, 1993; Toride et al., 1999; Zhang et al., 2006), although the practical implications of these distinctions are often not fully appreciated by many practicing engineers and scientists. The difference between resident and flux concentrations is illustrated in Fig. 1 for a well screened across zones with different groundwater velocities and local concentrations (e.g., due to nonequilibrium mass transfer). A water sample obtained from the well using usual field methods would approximate a flowweighted average ("flux concentration") along the length of the well screen. Alternatively, a water sample extracted from a composite soil sample over the same depth interval would yield a volume-weighted average ("resident concentration"). For the hypothetical example in Fig. 1, which corresponds to aquifer flushing after the primary contaminant source has largely dissipated, the average resident concentration over the well bore length is 17 times greater than the flux concentration due slow back-diffusion from the lower permeability zone.

If we wish to model such heterogeneous systems implicitly as "equivalent" mono-continua, transport equations that clearly distinguish between resident and flux concentrations must be formulated. In the following, we expand the 1-D nonreactive derivation of Kreft and Zuber (1978) and others to consider 3-D reactive transport. The mass balance equation for resident concentration during steady-state flow for this case is

$\phi \mathrm{R} \frac{\partial C_{R}}{\partial t}=-\nabla \mathbf{J}-\lambda \phi C_{R}$

where $C_{R}$ is resident concentration (mass of aqueous phase solute per unit fluid volume in the aquifer), $\phi$ is the total porosity $\left[\mathrm{L}^{3} \mathrm{~L}^{-3}\right], R$ is a retardation coefficient for linear sorption $\left[\mathrm{L}^{0}\right], \mathrm{J}$ is a solute mass flux density vector $\left[\mathrm{M} \mathrm{L}^{-2} \mathrm{~T}^{-1}\right], \lambda$ is a first-order decay coefficient $\left[\mathrm{T}^{-1}\right]$, and $\nabla$ is the spatial differentiation operator. The mass flux equation is assumed to be of the form

$\mathbf{J}=\mathbf{q} C_{R}-\phi \mathbf{D} \nabla C_{R}$

where $\mathbf{D}$ is an "effective" dispersion tensor for combined effects of diffusion and hydrodynamic dispersion $\left[\mathrm{L}^{2} \mathrm{~T}^{-1}\right]$, and $\mathbf{q}$ is the Darcy velocity vector $\left[\mathrm{L} \mathrm{T}^{-1}\right]$. The resulting mADE in terms of resident concentration from Eqs. (1) and (2) is

$R \frac{\partial C_{R}}{\partial t}=\mathbf{D} \nabla^{2} C_{R}-\mathbf{v} \nabla C_{R}-\lambda C_{R}$

where $\mathbf{v}=\boldsymbol{b}^{-1} \mathbf{q}$ is the mean superficial pore velocity.

Flux concentration $C_{F}$ is the solute mass discharge per volume of fluid discharge on a control surface formally defined if the resultant of $\mathbf{q}>0$ by

$$
C_{F}=\mathbf{q}^{-T} \mathbf{J}
$$

which, in conjunction with Eq. (2), indicates,

$C_{F}=C_{R}-\mathbf{v}^{-T} \mathbf{D} \nabla C_{R}$

If molecular diffusion is small relative to hydrodynamic dispersion, Eq. (5a) simplifies to

$C_{F}=C_{R}-A_{L} \frac{\partial C_{R}}{\partial \chi}$ 


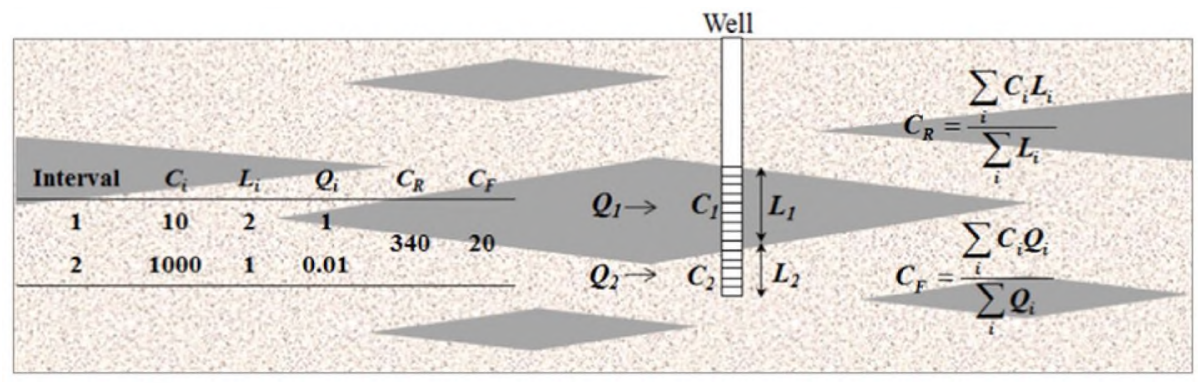

Fig. 1. lllustration of flow-averaged concentration $\left(C_{F}\right)$ observed in well screened over high and low permeability zones and volume-averaged concentration ( $\left.C_{R}\right)$ that would be determined in fluid extracted from adjacent soil samples aggregated over the same length.

where $A_{L}$ is longitudinal dispersivity [L] and $\chi$ is the local mean flow direction. Combining Eqs. (1) and (4) yields

$R \frac{\partial C_{R}}{\partial t}=-\mathbf{v}^{T} \nabla C_{F}-\lambda C_{R}$

and differentiating Eq. (5a) with respect to time gives.

$\frac{\partial C_{F}}{\partial t}=\frac{\partial C_{R}}{\partial t}-\mathbf{v}^{-T} \mathbf{D} \nabla \frac{\partial C_{R}}{\partial t}$

Using Eq. (6) to eliminate $C_{R}$ time derivatives from Eqs. (7) and (5a) to eliminate $C_{R}$ in decay terms yields a transport equation in terms of flux concentration

$R \frac{\partial C_{F}}{\partial t}=\mathbf{D} \nabla^{2} C_{F}-\mathbf{v} \nabla C_{F}-\lambda C_{F}$

Thus, the transport equation for flux concentrations has the identical mathematical form as the resident concentration mADE. However, correct solutions for the two formulations require a consistent application of boundary conditions. In particular, if the resident concentration $\mathrm{MADE}$ is solved for a specified mass flux from a contaminant source, solution of the corresponding flux concentration mADE must be obtained subject to a specified flux concentration $C_{F}=\mathrm{J} / \mathrm{q}$ to satisfy Eq. (4) where J and q are the scalar resultants of the boundary contaminant and water fluxes, respectively. The resulting solutions will thus obey the equalities given by Eqs. (4) and (5a). Alternatively, if a model is formulated and solved for resident concentrations, flux concentrations may be backcalculated from resident values by application of Eq. (5b).

One additional caveat must be considered in the formulation of boundary conditions to obtain physically meaningful mADE solutions for resident or flux concentrations in heterogeneous media. The effective dispersion tensor in Eq. (2) is generally assumed to have the form

$\mathbf{D}=D_{0} \boldsymbol{T}+\mathbf{v A}$

where $D_{0}$ is the (isotropic) molecular diffusion coefficient $\left[\mathrm{L}^{2} \mathrm{~T}^{-1} \mid, \tau\right.$ is a tortuosity tensor $\left[\mathrm{L}^{0}\right]$, and $\mathrm{A}$ is a hydrodynamic dispersivity tensor [L]. An inherent problem in Eq. (9) is that while molecular diffusion can occur in all directions independent of groundwater flow direction, hydrodynamic dispersion cannot occur upstream of the source location. This is so, because molecular diffusion is driven by chemical potential energy gradients, while hydrodynamic dispersion is driven physically by spatial variability in groundwater velocity, which is in turn controlled by the hydraulic gradient and local permeability variability. Spatial variability in hydraulic gradient direction may occur but will certainly not exceed $\pm 90^{\circ}$ from the mean direction. Therefore, the lower limit of spatial velocity variations will be zero. Negative velocities (i.e., flow opposite from the mean direction) may be precluded from consideration. Thus, if contaminants are released at a given location in a steady state flow field, while upstream diffusion may occur, hydrodynamic dispersion should be negligible.

We will refer here to the mathematical (but nonphysical) representation of hydrodynamic dispersion in a direction hydraulically upgradient of a contaminant source as "backdispersion." Note that for transient flow, the flow direction may change with time. If such fluctuations exceed $90^{\circ}$, backdispersion (relative to the mean flow direction) will be observed. Such cases should be simulated using a transient flow model to capture this effect. Also, in certain cases (i.e., localized water discharge at or near the source location that results in hydraulic mounding) water will flow and dispersion will occur locally "upstream" of the source relative to the regional flow direction. If such a problem is solved assuming a uniform regional flow field without consideration of mounding, some degree of apparent back-dispersion would occur, which will not be simulated accurately without consideration of mounding.

What are the mathematical consequences of allowing nonphysical back-dispersion? For simplicity assume an instantaneous pulse injection of contaminant. If longitudinal dispersivity is small relative to the plume length downstream from the source, back-dispersion will be negligible regardless of boundary conditions imposed. However, if dispersivity is much larger and back-dispersion is permitted, the simulated plume may extend far upgradient of the source location. The contaminant distribution along the longitudinal plume axis will be symmetric (normally distributed) about the peak concentration, which moves downstream at the mean transport velocity. Early breakthrough and long tails on breakthrough curves will not be predicted. What if backdispersion is mathematically disallowed? For a problem with a regional flow field without large transient variations in flow direction and without mounding near the source, this may be accomplished by treating the domain as semi-infinite in the mean flow direction starting at the source location. The semiinfinite solution can be extracted from the solution for a domain that extends upgradient of the source by imposing a reflection boundary perpendicular to flow at the source 
location. When the (nonphysical) upstream portion of the plume is "reflected" downstream, the center of mass will shift downstream (earlier breakthrough) and persist at a given location much longer (extended tailing), which are the characteristics we see in heterogeneous systems.

The correction for back-dispersion described above is equivalent to imposing a zero concentration gradient at the upstream edge of the source (or upstream of a water divide if water is also introduced at the source). This is also equivalent to setting $\mathbf{D}$ to zero immediately upgradient of the source. For multiple sources with overlapping plumes, linear analytical solutions for different sources may be superposed. For numerical models with multiple sources, placing a row of low dispersivity cells immediately upgradient of each source should prevent back-dispersion at source locations, while allowing upgradient plumes to continue downgradient by advection through the back-dispersion barrier.

A large body of theoretical and empirical research has shown that field-scale hydrodynamic dispersion is a manifestation of variability in contaminant velocity which increases with travel distance resulting in effective hydrodynamic dispersivities that increase with travel time when modeled as a Fickian process (e.g., Gelhar et al., 1992). In this context, the dispersion coefficient from Eq. (9) will be small near the source resulting in little back-dispersion. Thus, the back-dispersion problem is essentially a manifestation of the approximation of field-scale dispersion using a plume-averaged dispersivity. Unfortunately, this is by far the most common modeling approach in computer programs available to professionals working on field problems. There is a real need to develop methods to overcome these errors.

An additional characteristic of flux concentrations, which has received little attention, has important practical ramifications. For nonreactive 1-D transport, Kreft and Zuber (1978) and Zhang et al. (2006) noted that flux concentration is related to the rate of change of the integral of resident concentration change over space. A more general analysis for reactive 3-D transport indicates that the integral of flux concentration over a control plane depends on the rate of change in contaminant mass in all phases and the change due to reactions within the plume upgradient of the control plane (Fig. 2). The dependence of flux concentration on spatially integrated upstream transport processes implies that it will be much less sensitive to local heterogeneities in the vicinity of the sampling point than resident concentrations. This characteristic has important consequences for model calibration.

Despite the potentially large differences between resident and flux concentrations in heterogeneous media (Fig, 1), numerical models for groundwater transport are virtually always derived in terms of resident concentrations with no means to compute flux concentrations. Furthermore, unless the user takes precautions to avoid nonphysical back-dispersion, errors in computed resident concentrations can also occur. The advantage of numerical models is that if heterogeneities are explicitly treated at a sufficiently high resolution, resident and flux concentrations can be post-processed from model output at a scale consistent with measurements. However, this is not always feasible, due to high costs to characterize heterogeneities and/or execute a model at sufficiently high resolution. The situation is worse when commonly used 2-D and 3-D analytical transport models are used (e.g., Domenico, 1987; Galya, 1987; Wexler, 1992), which do not distinguish resident and flux concentrations, are subject to back-dispersion, and cannot explicitly treat heterogeneities.

In the following section, we present 3-D analytical solutions that compute resident or flux concentrations accurately even with very high dispersivities.

\subsection{Analytical 3-D solutions for resident and flux concentrations}

Numerous analytical solutions have been derived for one-, two- and three-dimensional transport problems based on the mADE for effectively homogeneous media (Ogata and Banks, 1961; Yeh and Tsai, 1976; Sagar, 1982; Cleary and Ungs, 1978; Domenico, 1987; Galya, 1987; Huyakorn et al., 1987; Leij and Dane, 1990; Leij et al., 1991; Wexler, 1992; Batu, 1996; Toride et al., 1999; Guyonnet and Neville, 2004; Falta et al., 2005) as well as for specific heterogeneous cases (Coats and Smith, 1964; Tang et al., 1981; Gillham et al., 1984; Barker, 1985; Starr et al., 1985; Sudicky et al., 1985; Barry and Parker, 1987; Liu

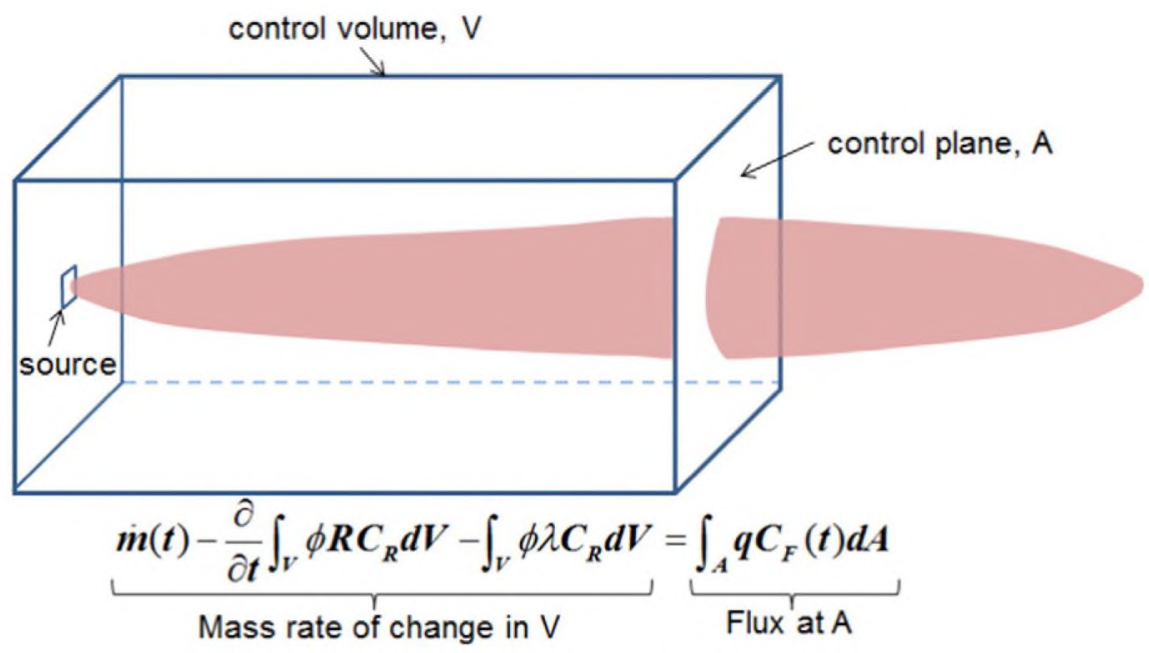

Fig. 2. Illustration of the dependence of flux concentration at a control plane on integral of upgradient transport processes. 
et al., 2000). Although subject to many simplifying assumptions, such solutions, when applicable, greatly reduce computational costs compared to numerical methods and provide useful baselines for numerical model verification.

In the following, we present 3-D solutions for resident and flux concentrations in an aquifer of finite thickness $L_{a q}$ with a steady-state uniform planar flow field in the $x$-direction with a contaminant source on vertical plane centered at $x=0, y=0$, $z=0$. The $x$-dimension is treated as infinite in the positive (downstream) direction, the $y$-direction is infinite, and the $z$-direction may be semi-infinite or finite as discussed later. For the assumed conditions, Eqs. (3) and (8) both simplify to

$\frac{\partial C}{\partial t}=A_{x} v \frac{\partial^{2} C}{\partial x^{2}}+A_{y} v \frac{\partial^{2} C}{\partial y^{2}}+A_{z} v \frac{\partial^{2} C}{\partial z^{2}}-v \frac{\partial C}{\partial x}-\lambda C$

where $A_{x}, A_{y}$ and $A_{z}$ are dispersivities [L] in longitudinal, transverse horizontal, and vertical directions, respectively; $x$ is distance from the source in the direction of flow [L]; $y$ is lateral distance from the center of the source perpendicular to flow $[\mathrm{L}] ; z$ is vertical distance $[\mathrm{L}] ; t$ is time since the initial release $[\mathrm{T}]$; and $\mathrm{C}\left[\mathrm{ML}^{-3}\right]$ may represent resident or flux concentration depending on the boundary conditions applied.

An arbitrarily time-dependent contaminant source with mass discharge rate $m(t)\left[\mathrm{M} \mathrm{T}^{-1}\right]$ distributed uniformly over a vertical plane perpendicular to flow of width $L_{y}$ and height $L_{z}$ with its center at $x=0$ and $y=0$. The location of $z=0$ depends on various cases described later. Zero concentration gradients for resident and flux solutions are assumed in the $z$-direction to the top and bottom of the aquifer, in the $y$-direction at $y= \pm \infty$, and in the $x$-direction at $x=+\infty$ and at $x=0$ other than vertical plane source area. Note that the boundary conditions at $x=0$ preclude upstream migration of contaminant. For the $C_{R}$ solution of Eq. (10a), the mathematical boundary condition on the source plane area is

$\mathrm{q}\left(C_{R}-A_{L} \frac{\partial C_{R}}{\partial x}\right)=\mathrm{J}(t)$

where $J(t)=m(t) / L_{y} L_{z}$. For the $C_{F}$ solution, Eq. (10b) transforms via Eqs. (5a) and (5b) to.

$\mathrm{q} C_{F}=\mathrm{J}(t)$.

The solution of Eqs. (10a)-(10c) for an arbitrary contaminant mass discharge rate as a function of time can be obtained by convolution as

$C(x, y, z, t)=\frac{1}{L_{y} L_{z} \phi} \int_{0}^{t} \dot{m}(\tau) f_{x}(x, t-\tau) f_{y}(y, t-\tau) f_{z}(z, t-\tau) \mathrm{d} \tau$

where $\tau$ is a dummy time integration variable $[T], f_{\mathcal{X}}(x, t)$ is the solution in the $x$-direction for a Dirac pulse (i.e., instantaneous unit mass injection) $\left[\mathrm{L}^{-1}\right], f_{y}(y, t)$ is the dispersion solution in the $y$-direction $\left[\mathrm{L}^{0}\right]$, and $f_{z}(z, t)$ is the $z$-direction dispersion solution $\left[\mathrm{L}^{0}\right]$.

Toride et al. (1999, Table 2.2) present 1-D solutions for instantaneous unit mass injection in time for resident and flux concentrations for an unbounded solution domain in the positive $x$-direction. Dispersion is not allowed upstream of the source. Solutions for $f_{x}(x, t)$ are

$$
\begin{aligned}
f_{x}^{F}(x, \tau)= & \frac{R x}{v \tau\left(4 \pi R A_{x} v \tau\right)^{1 / 2}} \exp \left(\frac{-\lambda \tau}{R}-\frac{(R x-v \tau)^{2}}{4 R A_{x} v \tau}\right) \\
f_{x}^{R}(x, \tau)= & \exp \left(\frac{-\lambda \tau}{R}\right) \\
& \times\left\{\begin{array}{l}
\frac{1}{\left(\pi A_{x} v R \tau\right)^{1 / 2}} \exp \left(-\frac{(R x-v \tau)^{2}}{4 A_{x} v R \tau}\right) \\
-\frac{1}{2 A_{x} R} \exp \left(\frac{x}{A_{x}}\right) \operatorname{erfc}\left(\frac{R x+v \tau}{\left(4 A_{x} v R \tau\right)^{1 / 2}}\right)
\end{array}\right\}
\end{aligned}
$$

where $f_{x}^{R}$ and $f_{x}^{E}$ are functions for resident and flux solutions, respectively. An alternative formulation in lieu of Eq. (11), which can be integrated more efficiently for locations near the source, is

$C(x, y, z, t)=\frac{1}{L_{y} L_{z} q} \int_{0}^{\dot{m}(t)} g_{x}(x, t-\tau) f_{y}(y, t-\tau) f_{z}(z, t-\tau) \mathrm{d} m(\tau)$

where $g_{x}(x, t)$ represents the solution for continuous injection at a constant unit rate. Note that since $m(t)$ is a function of time, numerical integration of Eqs. (10a)-(10c) can be performed either by (i) integrating over a specified $\Delta t$ and computing $\Delta m(t)$ corresponding to each time-step using Eq. (11), or (ii) integrating over a specified $\Delta m(t)$ and back-calculating $t$ for each step for use in $g_{x}$ and other terms in Eq. (13). Resident and flux solutions for $g_{x}$ are

$$
\begin{aligned}
g_{x}^{F}(x, \tau)= & \frac{1}{2} \exp \left(\frac{(v-u) x}{2 A_{x} v}\right) \operatorname{erfc}\left(\frac{R x-u \tau}{2\left(R A_{x} v \tau\right)^{1 / 2}}\right) \\
& +\frac{1}{2} \exp \left(\frac{(v+u) x}{2 A_{x} v}\right) \operatorname{erfc}\left(\frac{R x+u \tau}{2\left(R A_{x} v \tau\right)^{1 / 2}}\right)
\end{aligned}
$$

$$
\begin{aligned}
g_{x}^{R}(x, \tau)= & \frac{1}{v+u} \exp \left(\frac{(v-u) x}{2 A_{x} v}\right) \operatorname{erfc}\left(\frac{R x-u \tau}{2\left(R A_{x} v \tau\right)^{1 / 2}}\right) \\
& +\frac{1}{v-u} \exp \left(\frac{(v+u) x}{2 A_{x} v}\right) \operatorname{erfc}\left(\frac{R x+u \tau}{2\left(R A_{x} v \tau\right)^{1 / 2}}\right) \\
& +\frac{v}{2 \lambda A_{x} v} \exp \left(\frac{v x}{A_{x} v}-\frac{\lambda \tau}{R}\right) \operatorname{erfc}\left(\frac{R x+v \tau}{2\left(R A_{x} v \tau\right)^{1 / 2}}\right) \quad \text { for } \lambda>0 \\
g_{x}^{R}(x, \tau)= & \frac{1}{2} \operatorname{erfc}\left(\frac{R x-v \tau}{2\left(R A_{x} v \tau\right)^{1 / 2}}\right)+\left(\frac{v^{2} \tau}{\pi R A_{x} v}\right)^{1 / 2} \exp \left(-\frac{(R x-v \tau)^{2}}{4 R A_{x} v \tau}\right) \\
& -\frac{1}{2}\left(1+\frac{v x}{A_{x} v}+\frac{v^{2} \tau}{R A_{x} v}\right) \exp \left(\frac{v x}{A_{x} v}\right) \operatorname{erfc}\left(\frac{R x+v \tau}{2\left(R A_{x} v \tau\right)^{1 / 2}}\right) \text { for } \lambda=0
\end{aligned}
$$

where $g_{x}^{R}$ and $g_{x}^{F}$ are functions for resident and flux concentrations $\left[\mathrm{L}^{0}\right]$, respectively, and $u=\left(v^{2}+4 \lambda A_{x} v\right)^{1 / 2}$. It may be noted that Eq. (12a) and (12b) will yield nearly identical results (hence $C_{R} \approx C_{F}$ ) when $A_{x} / x \ll 1$. This is also the case for Eqs. (14a) and (14b). 
The horizontal spreading terms in Eqs. (11) and (13) for an aquifer unbounded laterally is given by

$f_{y}(y, \tau)=\frac{1}{2}\left[\operatorname{erf}\left(\frac{|y|+L_{y} / 2}{2\left(A_{y} v \tau / R\right)^{1 / 2}}\right)-\operatorname{erf}\left(\frac{|y|-L_{y} / 2}{2\left(A_{y} v \tau / R\right)^{1 / 2}}\right)\right]$.

Note that symmetry about the center line yields the same function values for positive or negative $y$-values having the same absolute magnitude.

To account for different vertical source configurations, three conditions are distinguished for vertical dispersion terms as described below.

Condition 1. Fully penetrating source (2-D solution).

If the source thickness, $L_{z}$, is equal to the aquifer thickness, $L_{a q}$, then a 2-D solution strictly applies for which $f_{z}(z, t)=1$. A 2-D solution may also be applicable at distances sufficiently far downstream from the source that vertical dispersion yields essentially uniform mixing over the aquifer thickness. Condition 2. Partially penetrating source adjacent to top or bottom aquifer boundary.

For this condition, the top of the source zone may coincide with the aquifer upper boundary defined at $z=0$ (e.g., water table) and the bottom of the source is substantially above the aquifer lower boundary with $z$ increasing downwards. Alternatively, the bottom of the source coincides with the aquifer lower boundary defined locally as $z=0$ with $z$ increasing upwards.

The Condition 2 vertical dispersion function for an aquifer of infinite thickness is given by

$f_{z}^{\infty}(z, \tau)=\frac{1}{2}\left[\operatorname{erf}\left(\frac{z+L_{z}}{2\left(A_{z} v \tau / R\right)^{1 / 2}}\right)-\operatorname{erf}\left(\frac{z-L_{z}}{2\left(A_{z} v \tau / R\right)^{1 / 2}}\right)\right]$

An image boundary technique (e.g., Galya, 1987) is employed to correct for effects of plume interaction with finite vertical boundaries as follows

$$
\begin{aligned}
f_{z}(z, \tau)= & f_{z}^{\infty}(z, \tau)+f_{z}^{\infty}\left(2 L_{a q}-z, \tau\right)+f_{z}^{\infty}\left(2 L_{a q}+z, \tau\right) \\
& +f_{z}^{\infty}\left(4 L_{a q}-z, \tau\right)+f_{z}^{\infty}\left(4 L_{a q}+z, \tau\right) \\
& +f_{z}^{\infty}\left(6 L_{a q}-z, \tau\right)+f_{z}^{\infty}\left(6 L_{a q}+z, \tau\right)+\ldots
\end{aligned}
$$

Three subsidiary conditions may be distinguished for computing the Condition 2 dispersion function:

Condition 2a. $x<x_{\infty}=\left(L_{a q}-L_{z}\right)^{2} F_{\infty} / A_{z}$.

At distances closer than $x_{\infty}$ from the source, the plume has not intercepted the opposite aquifer boundary opposite the source. Therefore, no correction terms need to be applied and Eq. (16a) alone can be used to compute $f_{z}(z, \tau)=f_{z}(z$, $\tau)$. The factor $F_{\infty}=0.001$ was determined by comparing 3-D solution results with and without correction terms. Condition $2 b . x>x_{2 D}=\left(L_{a q}-L_{z}\right)^{2} F_{2 D} / A_{z}$.

At distances greater than $x_{2 D}$ from the source, vertical mixing yields negligible vertical concentration gradients and a 2-D approximation may be adopted as described above with $f_{z}(z, t)=1$. The factor $F_{2 D}=10$ was determined by comparing 3-D and 2-D solution results.

Condition $2 c . x_{\infty}<x<x_{2 D}$.

For this case, Eq. (16b) is employed using a sufficient number of correction terms to obtain desired accuracy. After computing the first two terms in Eq. (16b), the series is terminated when the last computed term is less than $0.1 \%$ of the sum of all terms computed to that point.

Condition 3. Source NOT immediately adjacent to top or bottom of aquifer.

Finally, we consider the instance in which the source does not lie immediately adjacent to the top or bottom of the aquifer. The center of the source of thickness $L_{z}$ is located at an elevation denoted locally as $z=0$ with positive $z$ above the source and negative below. The top of the source is at $z=L_{z} / 2$ and the aquifer upper boundary is at $z=L_{u}>L_{z} / 2$. The bottom of the source is at $z=-L_{z} / 2$ and the aquifer lower boundary is at $z=-L_{b}<-L_{z} / 2$. The vertical dispersion function for an unbounded aquifer is

$f_{z}^{\infty}(z, \tau)=\frac{1}{2}\left[\operatorname{erf}\left(\frac{|z|+L_{z} / 2}{2\left(A_{z} v \tau / R\right)^{1 / 2}}\right)-\operatorname{erf}\left(\frac{|z|-L_{z} / 2}{2\left(A_{z} v \tau / R\right)^{1 / 2}}\right)\right]$.

Note that although the function defined by Eq. (17a) is symmetric about $z=0$, corrections for finite aquifer thickness will be asymmetric unless the source lies exactly midway between the upper and lower aquifer boundaries. If corrections for finite aquifer thickness are required and $L_{u}-|z|<L_{b}-|z|$, the series with terms in order of decreasing magnitude is

$$
\begin{aligned}
f_{z}(z, \tau)= & f_{z}^{\infty}(z, \tau)+f_{z}^{\infty}\left(2 L_{b}+z, t\right)+f_{z}^{\infty}\left(2 L_{u}-z, t\right) \\
& +f_{z}^{\infty}\left(2 L_{b}+2 L_{u}-z, t\right)+f_{z}^{\infty}\left(2 L_{u}+2 L_{b}+z, t\right) \\
& +f_{z}^{\infty}\left(4 L_{b}+2 L_{u}+z, t\right)+f_{z}^{\infty}\left(4 L_{u}+2 L_{b}-z, t\right) \\
& +f_{z}^{\infty}\left(4 L_{b}+4 L_{u}-z, t\right)+f_{z}^{\infty}\left(4 L_{u}+4 L_{b}+z, t\right) \\
& +f_{z}^{\infty}\left(6 L_{b}+4 L_{u}+z, t\right)+f_{z}^{\infty}\left(6 L_{u}+4 L_{b}-z, t\right) \\
& +f_{z}^{\infty}\left(6 L_{b}+6 L_{u}-z, t\right)+f_{z}^{\infty}\left(6 L_{u}+6 L_{b}+z, t\right)+\ldots
\end{aligned}
$$

otherwise, the computational sequence is

$$
\begin{aligned}
f_{z}(z, t)= & f_{z}^{\infty}(z, t)+f_{z}^{\infty}\left(2 L_{u}-z, t\right)+f_{z}^{\infty}\left(2 L_{b}+z, t\right) \\
& +f_{z}^{\infty}\left(2 L_{u}+2 L_{b}+z, t\right)+f_{z}^{\infty}\left(2 L_{b}+2 L_{u}-z, t\right) \\
& +f_{z}^{\infty}\left(4 L_{u}+2 L_{b}-z, t\right)+f_{z}^{\infty}\left(4 L_{b}+2 L_{u}+z, t\right) \\
& +f_{z}^{\infty}\left(4 L_{u}+4 L_{b}+z, t\right)+f_{z}^{\infty}\left(4 L_{b}+4 L_{u}-z, t\right) \\
& +f_{z}^{\infty}\left(6 L_{u}+4 L_{b}-z, t\right)+f_{z}^{\infty}\left(6 L_{b}+4 L_{u}+z, t\right) \\
& +f_{z}^{\infty}\left(6 L_{u}+6 L_{b}-z, t\right)+f_{z}^{\infty}\left(6 L_{b}+6 L_{u}+z, t\right)+\ldots
\end{aligned}
$$

The series is truncated after the last computed term is less than $0.1 \%$ of the sum of all terms to that point. Sub- 
conditions analogous to those for Condition 2 may be distinguished as follows.

Condition 3a. If $x<x_{\infty}=\left(L_{\min }-L_{z} / 2\right)^{2} F_{\infty} / A_{z}$ where $L_{\min }=$ $\min \left(L_{u}, L_{b}\right)$ then Eq. (17a) with no corrections is used to compute $f_{z}(z, \tau)=f_{z}^{\circ}(z, \tau)$.

Condition $3 b$. If $x>x_{2 D}=\left(L_{\max }-L_{z} / 2\right)^{2} F_{2 D} / A_{z}$ where $L_{\text {max }}=\max \left(L_{u}, L_{b}\right)$ then a 2-D approximation with $f_{z}(z, t)=$ 1 is employed.

Condition 3c. If $x_{\infty}<x<x_{2 D}$ then Eq. (17b) or Eq. (17c) is used to compute correction terms until the truncation criteria is met.

The solutions described above have been implemented in the MATLAB-based Stochastic Cost Optimization Toolkit (SCOToolkit) program, which couples the transport model with various remediation technology simulators, an inverse solution for model calibration, a cost model, a Monte Carlo algorithm, and a stochastic optimization algorithm for remediation system design (Parker et al., 2011). The program also implements a coordinate transformation scheme to simulate transport in mildly curvilinear flow fields and superposition methods to model multiple DNAPL sources and electron-donor limited decay. The program is available on request from the authors.

\subsection{Comparison of analytical and mumerical solutions for resident and flux concentrations}

We will compare results for the above analytical solutions with the widely used MT3D finite difference code to verify correctness of the analytical model derivations and implementation and to evaluate methods to obtain resident and flux concentration solutions using numerical models when the source cannot be located at the upstream model boundary.

To obtain a resident concentration solution from MT3D or any other numerical model, attention needs to be given to the following issues:

1. A contaminant release event may be modeled by specifying the source mass discharge rate versus time directly, or if contaminant is introduced in conjunction with water recharge, the hydraulic recharge rate and recharge concentration may be given. In many instances, no attempt to model release events is made and the contaminant plume existing at a certain date is specified by giving initial conditions based on field measurements. This approach is valid provided field data in fact reflect resident concentrations. However, most field measurements are obtained from well samples, which should be regarded as flux concentrations. Refer to options for simulating flux concentrations below.

2. No hydrodynamic dispersion upgradient of the source must be allowed. This can be easily accomplished for a single source with negligible associated hydraulic loading by placing the source at the upgradient model boundary with a zero concentration gradient imposed (this is the natural condition which requires no user input for most numerical models). If the source must be located downstream of the upgradient boundary, model cells immediately upstream of the source should be assigned a low dispersivity to limit upstream dispersion. If hydraulic discharge at or near the source results in mounding at the source, upgradient dispersion may be allowed up to the flow divide.

3. The model boundary should extend beyond the maximum extent of the plume for consistency with the condition of zero gradient at $x=+\infty$.

Two options are considered to obtain numerical solutions for flux concentrations:

1. Solve for $C_{R}$ first as described above and then post-process model output to compute $C_{F}$ values at locations and times of interest by application of Eqs. (5a) and (5b). This requires numerical evaluation of the gradient of $C_{R}$ in the local direction of flow.

2. Solve the flux-transformed form of the mADE to obtain $C_{F}$ directly. Initial conditions must be specified based on flux concentration measurements (e.g., monitoring wells). If resident concentration data are available to define initial conditions, they may be transformed to flux concentrations first by application of Eqs. (5a) and (5b). If release events are to be modeled, the source mass discharge rate must be transformed to a flux concentration boundary condition. For the vertical plane source considered in the analytical model, the transformation is

$C_{0}(t)=\frac{m(t)}{q L_{y} L_{z}}$

where $C_{0}(t)$ is the flux concentration boundary condition versus time. This second option has certain limitations that will be discussed later.

\subsection{Interpretation of the $M A D E$ as an upscaled model for diffusion-limited mass transfer}

Many mathematical models have been presented for transport in media characterized by "mobile" regions in which contaminants move primarily by advection and "immobile" regions where contaminants move primarily by diffusion. We consider here the interpretation of resident and flux solutions of the mADE, such as described above, in terms of a bi-continuum mobile-immobile model quantified by the following parameters:

$q_{m} \quad$ mobile zone Darcy velocity $\left[\mathrm{L} \mathrm{T}^{-1}\right]$.

$F_{m} \quad$ ratio of mobile zone volume to total aquifer volume $\left[\mathrm{L}^{3} \mathrm{~L}^{-3}\right]$

$A_{m} \quad$ mobile zone longitudinal dispersivity [L].

$L_{i m} \quad$ average immobile zone diffusion path length [L]

$D_{i m} \quad$ immobile zone effective molecular diffusion coef ficient $\left[\mathrm{L}^{2} \mathrm{~T}^{-1}\right]$

$\phi_{m} \quad$ mobile zone water volume as fraction of total mobile zone volume $\left[\mathrm{L}^{3} \mathrm{~L}^{-3}\right]$

$\phi_{i m} \quad$ immobile zone water volume as fraction of total immobile zone volume $\left[\mathrm{L}^{3} \mathrm{~L}^{-3}\right]$

$\rho_{I m} \quad$ dry soil mass in mobile zone as fraction of total mobile zone volume $\left[\mathrm{M} \mathrm{L}^{-3}\right.$

$\rho_{i m} \quad$ dry soil mass in immobile zone as fraction of total immobile zone volume $\left[\mathrm{M} \mathrm{L}^{-3}\right]$

$k_{m} \quad$ mobile zone adsorption coefficient $\left[\mathrm{L}^{3} \mathrm{M}^{-1}\right]$

$k_{i m} \quad$ immobile zone adsorption coefficient $\left[\mathrm{L}^{3} \mathrm{M}^{-1}\right]$ 
$\lambda_{m} \quad$ mobile zone first-order decay coefficient $\left[\mathrm{T}^{-1}\right]$

$\lambda_{i m} \quad$ immobile zone first-order decay coefficient $\left[\mathrm{T}^{-1}\right]$.

A number of studies (Bolt, 1979; Passioura, 1971; Raats, 1981; Parker and Valocchi, 1986; van Genuchten, 1985; van Genuchten and Dalton, 1986) have shown that diffusionlimited mobile-immobile model solutions can be approximated by simple mono-continuum dispersion models using "effective" (upscaled) parameters given by

$A_{e f f}=f_{m} A_{m}+\frac{\left(1-f_{m}\right) v L_{i m}^{2} R_{i m}^{2}}{4 \gamma D_{i m} R^{2}}$

$v=\frac{q}{\phi}=\frac{F_{m} q_{m}}{\phi}$

$\phi=F_{m} \phi_{m}+\left(1-F_{m}\right) \phi_{i m}$

$f_{m}=\frac{F_{m} \phi_{m}}{F_{m} \phi_{m}+\left(1-F_{m}\right) \phi_{i m}}$

$\rho=F_{m} \rho_{m}+\left(1-F_{m}\right) \rho_{i m}$

$R=f_{m} R_{m}+\left(1-f_{m}\right) R_{i m}$

$R_{m}=1+\frac{\rho_{m} k_{m}}{F_{m} \phi_{m}}$

$R_{i m}=1+\frac{\rho_{i m} k_{i m}}{\left(1-F_{m}\right) \phi_{i m}}$

$\lambda=f_{m} \lambda_{m}+\left(1-f_{m}\right) \lambda_{i m}$

where $A_{\text {eff }}$ is the effective longitudinal dispersivity, $v$ is the average aquifer pore velocity, $f_{m}$ is the mobile pore fraction, $\phi$ is the average aquifer porosity, $\rho$ is the average aquifer bulk density, $R$ is the effective retardation factor for the aquifer, $\lambda$ is the effective aquifer decay coefficient, and $\gamma$ is a geometry factor tabulated in Table 1 for various media configurations based on van Genuchten and Dalton (1986).

Using upscaled parameters given by Eqs. (19a)-(19i) in the mADE will yield first and second moments of flux concentra-

Table 1

Geometry factors for upscaled dispersion model (van Genuchten and Dalton, 1986).

\begin{tabular}{lll}
\hline Geometry of media & Geometry factor, $\gamma$ & $L_{i m}$ \\
\hline $\begin{array}{l}\text { Spherical aggregates in mobile } \\
\text { matrix }\end{array}$ & 15 & Sphere radius \\
$\begin{array}{l}\text { Solid cylindrical aggregates in } \\
\text { mobile matrix }\end{array}$ & 8 & Cylinder radius \\
Planar sheets in mobile matrix & 3 & $\begin{array}{l}\text { Sheet half- } \\
\text { thickness }\end{array}$ \\
$\begin{array}{l}\text { Hollow cylinders with } \\
\text { "wormhole"a }\end{array}$ & {$\left[0.5 \ln \left(\frac{b}{a}\right)-0.25\right]^{-1}$} & $b-a$
\end{tabular}

a $a=$ outer radius and $b=$ "wormhole" radius for hollow cylinder case. tion versus time for a Dirac injection equal to those for the bi-continuum model of specified fracture-matrix geometry (Parker and Valocchi, 1986).

To the extent mobile-immobile model parameters can be directly estimated from field and lab data, the foregoing relationships can be used to estimate (upscaled) mADE parameters, which can then be refined, if necessary, by calibration to field data. Potential types of field data and their interpretations include the following.

\subsubsection{Monitoring well data}

Dissolved concentrations from well samples should be regarded as upscaled model flux concentrations, $C_{F}$. This is true regardless of whether immobile zones occur or not, since immobile zones, by definition, do not contribute to flux concentration.

\subsubsection{Small-scale dissolved concentration samples}

If dissolved concentration samples are obtained using suction devices (e.g., in conjunction with geoprobe equipment) at a scale smaller than the spacing between mobile and immobile zones, the values can best be interpreted as local scale resident concentrations. To obtain average resident concentrations, $C_{R}$, at a scale that encompasses mobile and immobile zones, local concentrations within an appropriate distance can be averaged and used for calibration. Alternatively, local values may be used directly for calibration using a least squares objective. However, residual deviations will be much larger due to the scale mismatch.

\subsubsection{Soil concentration data averaged over mobile and immobile zones}

If the spacing between mobile and immobile zones is small compared to the length over which soil samples are taken, measurements of total soil concentration (dissolved and sorbed contaminant mass per dry soil mass, $S_{a l l}$ ) can be interpreted as

$S_{\text {all }}=\frac{R \phi C_{R}}{\rho}$

where all variables represent upscaled model values.

\subsubsection{Soil concentrations from mobile zone samples}

If soil samples are taken from identifiable mobile zones with in the aquifer, measured mobile zone soil concentrations, $S_{m}$, can be interpreted as

$S_{m}=\frac{R_{m} \phi_{m} C_{F}}{\rho_{m}}$

assuming that the mobile zone dissolved concentration is approximately equal to the upscaled model flux concentration since mobile zone dispersivity is generally small. The aqueous + sorbed mobile zone contaminant mass per total (mobile + immobile zone) soil mass, $S_{m / t}$, is

$S_{m / t}=f_{m} R_{m} \phi_{m} C_{F}$ 
2.4.5. Soil concentrations from immobile zone samples

If soil samples are taken from identifiable low permeability zones, the measured immobile zone contaminant mass per dry mass of immobile zone soil, $S_{i m}$, can be interpreted as

$S_{i m}=\frac{\rho S_{\text {all }}-f_{m} \rho_{m} S_{m}}{\left(1-f_{m}\right) \rho_{i m}}$

while the aqueous + sorbed immobile zone contaminant mass per total (mobile + immobile zone) soil mass, $S_{i m / t}$, can be interpreted as

$S_{i m / t}=\rho S_{\text {all }}-f_{m} \rho_{m} S_{m}$

Note that interpretation of mobile and immobile zone soil concentrations depends on both upscaled and bi-continuum model parameters.

\section{Results and discussion}

\subsection{Comparison of analytical and numerical solutions}

In this section we present results of simulations using the analytical model described in Section 2.2 and the numerical model MT3D for various cases (Table 2). In addition to verifying the analytical model implementation with a well-established numerical model, our objectives are to:

- evaluate differences between solutions for resident and flux concentrations

- assess consequences of misinterpreting $C_{R}$ solutions as $C_{F}$ or vise versa

- evaluate various methods to compute $C_{R}$ and $C_{F}$ using a numerical model

- assess effects of up- and downstream boundary conditions on numerical model accuracy.

The example 1-D problems assume a contaminant source with a discharge rate $m=0.01 \mathrm{~kg} / \mathrm{d} / \mathrm{m}^{2}$ of source area for 15 years followed by $m=0$ for an additional 135 years. The Darcy velocity is $0.1 \mathrm{~m} / \mathrm{d}$, porosity is 0.3 , dispersivity is $200 \mathrm{~m}$, the retardation factor is 10 , and the first-order decay coefficient is $0.002 \mathrm{~d}^{-1}$. Molecular diffusion is disregarded. From Eq. (18), the flux concentration boundary condition at the source is $C_{0}=100,000 \mu \mathrm{g} / \mathrm{L}$ for the first 15 years and $C_{0}=0$ thereafter.
Grid spacing in the $x$-direction for numerical simulations was $10 \mathrm{~m}$ for the first $1000 \mathrm{~m}$ travel distance and $20 \mathrm{~m}$ thereafter. For 3-D simulations, grid spacing was $5 \mathrm{~m}$ from $y=0$ (centerline) to $y=15 \mathrm{~m}, 10 \mathrm{~m}$ spacing from $y=15 \mathrm{~m}$ to $y=75 \mathrm{~m}$, and $1 \mathrm{~m}$ spacing in the $z$-direction.

A comparison of simulated resident and flux concentrations versus time at $x=100$ and $500 \mathrm{~m}$ is shown in Fig. 3 for numerical and analytical solutions. The model domain was extended from the source location (i.e., $x=0$ ) to $3000 \mathrm{~m}$ downgradient. Upgradient dispersion is effectively eliminated since there is no domain upgradient of the source. The numerical solution for resident concentration (Case 1a, Table 1) uses a mass flux boundary condition ("constant head" boundary condition in MT3D). Two numerical solutions for flux concentration are shown. Case 1b computes flux concentrations by applying Eq. (5b) to Case 1a resident concentrations, while Case 2 computes $C_{F}$ directly using a concentration boundary condition ("constant concentration" boundary condition in MT3D) given by Eq. (18). The downstream boundary for all of the foregoing numerical simulations is located $3000 \mathrm{~m}$ from the source, except Cases 4 and 5 in Table 2.

In Fig. 3, analytical and numerical solutions for $C_{R}$ are in close agreement with each other, as are the analytical and both numerical solutions for $C_{F}$. Values of $C_{f}$ are greater than $C_{R}$ during the source release period, while the converse is true after source removal. With a retarded plume velocity of $12.2 \mathrm{~m} /$ year, the mean travel time to reach $100 \mathrm{~m}$ is about 8 years. However, breakthrough occurs in less than 1 year and tailing continues for 90 years due to nonequilibrium mass transfer between mobile and immobile zones. Results at $500 \mathrm{~m}$ exhibit similar, but somewhat muted, behavior.

The cumulative mass passing a given distance, $x$, can be computed by integrating $q C_{Y}(x, t)$ over time. Carrying out this integration for the numerical and analytical $C_{F}$ solutions yield mass estimates that agree within $\sim 3 \%$. However, if a $C_{R}$ solution were erroneously interpreted as a $C_{F}$ solution, integration would underestimate the mass passing the monitoring point by $\sim 40 \%$.

Resident and flux concentrations versus distance at $t=25$ and 50 years are shown in Fig. 4 for the same cases described above. Good agreement is observed between the numerical and analytical solutions for $C_{R}$ and $C_{F}$, respectively, except leading edges for the numerical solutions are slightly advanced, which may be attributed to numerical dispersion. At a mean retarded

Table 2

Summary of numerical simulations.

\begin{tabular}{|c|c|c|c|c|c|c|}
\hline Case $^{a}$ & Model dimension & Output concentration type & Source boundary condition & Upstream dispersion? & $\begin{array}{l}\text { Distance to downstream } \\
\text { boundary }\end{array}$ & Post-process \\
\hline 1a & $1-\mathrm{D}$ & $C_{R}$ & $\mathrm{~J}(t)$ & No & $3000 \mathrm{~m}$ & No \\
\hline $1 \mathrm{~b}$ & $1-\mathrm{D}$ & $C_{F}$ & $\mathrm{~J}(t)$ & No & $3000 \mathrm{~m}$ & $C-A_{x} \mathrm{~d} C / \mathrm{d} x$ \\
\hline 2 & $1-D$ & $C_{F}$ & $C(t)$ & No & $3000 \mathrm{~m}$ & No \\
\hline $3 a$ & $1-\mathrm{D}$ & $C_{F}$ & $C(t)$ & Yes & $3000 \mathrm{~m}$ & No \\
\hline $3 b$ & $1-D$ & $C_{F}$ & $C(t)+$ no $B C^{b}$ & Yes & $3000 \mathrm{~m}$ & No \\
\hline 4 & $1-\mathrm{D}$ & $C_{F}$ & $C(t)$ & No & $100 \mathrm{~m}$ & No \\
\hline 5 & $1-\mathrm{D}$ & $C_{F}$ & $C(t)$ & No & $250 \mathrm{~m}$ & No \\
\hline $6 a$ & $3-D$ & $C_{R}$ & $\mathrm{~J}(t)$ & No & $3000 \mathrm{~m}$ & No \\
\hline $6 b$ & $3-D$ & $C_{F}$ & $\mathrm{~J}(t)$ & No & $3000 \mathrm{~m}$ & $C-A_{x} \mathrm{~d} C / \mathrm{d} x$ \\
\hline
\end{tabular}

\footnotetext{
a All cases release a source from $t=0$ to $t=15$ years.

b Specified concentration during release followed by no boundary condition.
} 

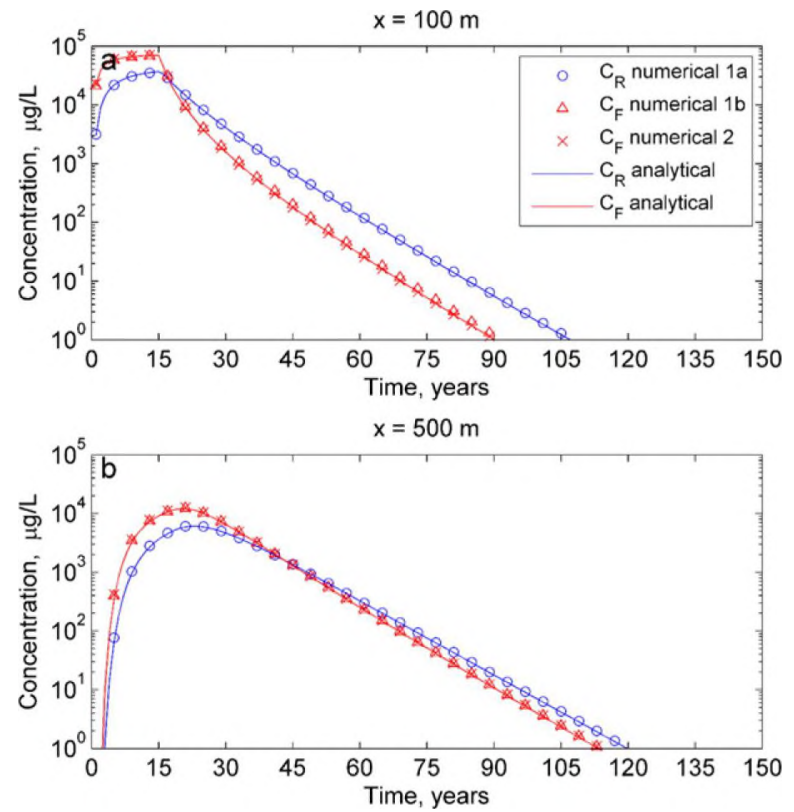

Fig. 3. Numerical and analytical simulations of resident and flux concentrations versus time at $x=100$ (a) and $500 \mathrm{~m}$ (b).

pore velocity of $12.2 \mathrm{~m} / \mathrm{year}$, the piston front location would be at about $300 \mathrm{~m}$ after 25 years and at $600 \mathrm{~m}$ after 50 years. At distances closer than this to the source, $C_{R}$ values exceed $C_{F}$ and at larger distanced the reverse is observed. Both $C_{R}$ and $C_{F}$ distributions are strongly skewed, with $1 \mu \mathrm{g} / \mathrm{L}$ front locations about 5 times ahead of the piston front location at 25 years and 3.8 times at 50 years. Since the contaminant source terminates after 15 years, $C_{F}=0$ at $x=0$ for both the 25 - and 50 -year plots. No contaminant occurs upgradient for either case, as constrained
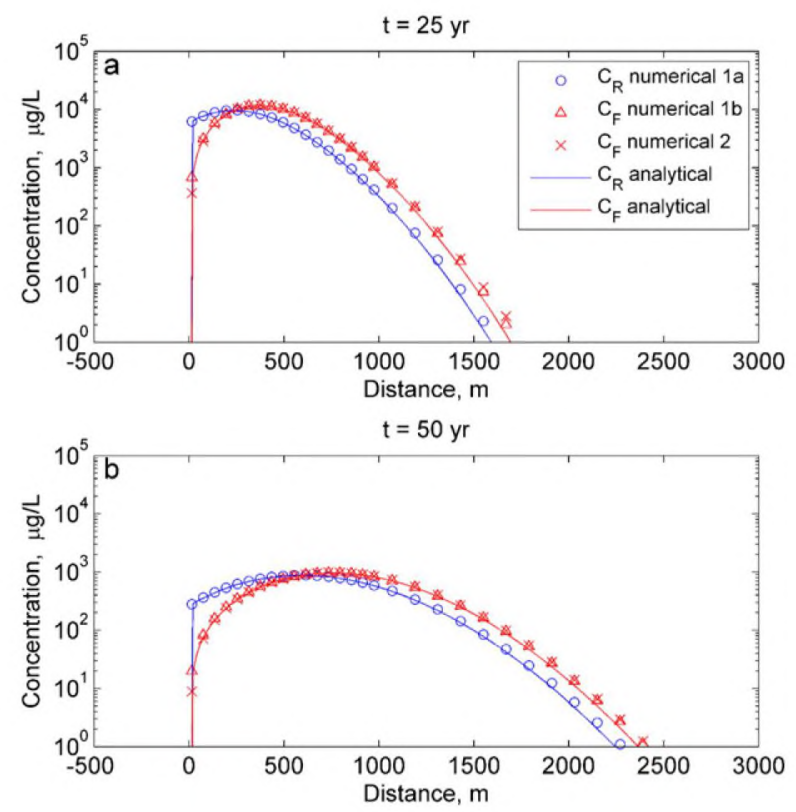

Fig. 4. Numerical and analytical 1-D simulations of resident and flux concentrations versus distance at $t=25$ (a) and 50 years (b). by imposed boundary conditions, however, $C_{R}$ exhibits a "jump" discontinuity at $x=0$. The magnitude of the jump in $C_{R}$ at $x=0$ may be shown to increase with longitudinal dispersivity.

It may be noted that an infinite resident concentration gradient at $x=0$ (or anywhere else) is not physically possible. Real plumes will exhibit more or less diffuse upstream boundaries as a result of molecular diffusion and spatially distributed sources. Both of these phenomena have been disregarded in the presented results. Although these simplifications are difficult to relax in an analytical model, both can easily be considered in numerical solutions while still precluding nonphysical upstream dispersion. In particular, the diffusion term in Eq. (9) can be included and only dispersivity set to zero for cells immediately upgradient of sources, and sources may be assigned to multiple cells in all three dimensions, as opposed to the vertical plane source assumed here. Horizontally distributed sources may be considered in analytical solutions at the expenses of some computational efficiency. The SCOToolkit solution incorporates an empirical correction for horizontally distributed sources that linearly interpolates the $x=0$ solution for $x<0$ to give $C_{R}=0$ at the specified upstream edge of the source defined as $x=-L_{x}$. This results in a slight mass balance error but provides more realistic behavior with negligible additional computational effort.

Total aqueous and sorbed mass in the aquifer at a given time may be computed by integrating $\phi R C_{R}$ over the aquifer volume. Carrying out this integration for the foregoing numerical and analytical $C_{R}$ solutions at 25 years yields a mass balance within $\sim 3 \%$. However, if the $C_{F}$ solution were erroneously interpreted as a $C_{R}$ solution, integration would overestimate the mass by $\sim 26 \%$.

Upstream dispersion in the above numerical simulations was prevented by placing the source at the upstream model boundary, which may not be a practical approach for many problems, especially when multiple sources occur. However, identical results were obtained using a model domain that extended upgradient of the source with zero dispersivity specified in cells immediately upgradient of the source.

Case 3 investigates the effect of upstream dispersion and source boundary conditions on numerical solution results using a model domain that extends $3000 \mathrm{~m}$ upgradient and downgradient of the source with a uniform dispersivity. Case 3a simulates $C_{F}$ using a boundary condition at the source of $C_{o}=100,000 \mu \mathrm{g} / \mathrm{L}$ during the release and $C_{o}=0$ thereafter. The breakthrough curve at $x=100 \mathrm{~m}$ agrees closely with the Case 2 numerical solution and the analytical solution (Fig. 5).

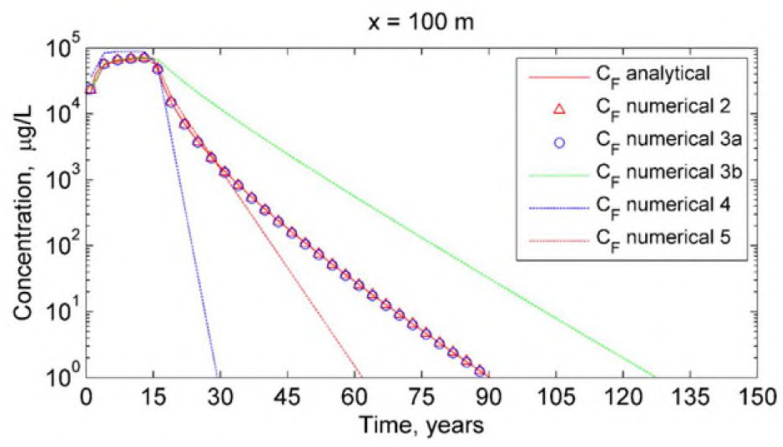

Fig. 5. Effects of upgradient and downgradient boundary conditions on 1-D numerical simulations of flux concentration versus time at $x=100 \mathrm{~m}$. 
However, inspection of simulated concentrations upgradient of the source (not shown) indicates a substantial plume upgradient of the source for Case $3 \mathrm{a}$, which should not occur. The $C_{o}=0$ boundary condition at the source effectively creates a sink-term at the source location, which prevents upgradient mass from passing $x=0$ after source removal.

The problem is clarified by the Case $3 \mathrm{~b}$ simulation, which is identical to Case 3a except that after source removal, a zero gradient rather than a zero concentration condition is imposed (no declared boundary condition in MT3D). In the absence of upgradient contaminant, the two conditions would be identical. However, Case 3b shows a more protracted breakthrough curve with a much greater total mass than that of the analytical and Case $1 \mathrm{~b}$ and Case 2 numerical solutions due to the upgradient plume associated with upgradient dispersion (Fig. 5).

Note that if multiple sources with overlapping plumes need to be simulated, imposing concentration boundary conditions at downgradient source locations would effectively create sinks for upgradient sources. Therefore, the most robust approach to simulate flux concentrations with a numerical model would be to solve directly for resident concentrations (using a specified flux source boundary condition) and to post-process resident concentrations to obtain flux concentrations using Eqs. (5a) or (5b).

The final 1-D problem considers the effect of downstream boundary conditions in a numerical model on solution accuracy. Cases 4 and 5 solve for flux concentrations with no upstream dispersion allowed, but with model domains that extend to only 100 and $250 \mathrm{~m}$, respectively, which are substantially shorter than the plume. With no explicit boundary condition specified at the downstream edge, MT3D assumes a zero concentration gradient. Tailing of the simulated breakthrough curves is sharply diminished as the model domain is increasingly truncated (Fig. 5). This occurs because the exit boundary condition allows only advective transport and no dispersion through the boundary. For very long plumes, it is not uncommon to truncate numerical model domains to focus on near-source areas of primary concern for remediation. It would be advisable to conduct sensitivity analyses in such cases to ensure that the domain is large enough to avoid unacceptable error.

3-D simulations were performed with MT3D and the analytical model using the same parameters used for 1-D simulations except that the source is $10 \mathrm{~m}$ wide, extends from the water table to a depth of $2.5 \mathrm{~m}$, and has a discharge rate of $0.2 \mathrm{~kg} / \mathrm{d}$ for 15 years followed by no discharge. The aquifer is $10 \mathrm{~m}$ thick with a transverse dispersivity of $1 \mathrm{~m}$ and a vertical dispersivity of $0.1 \mathrm{~m}$. Due to symmetry about the axis of the plume, only half of the plume was modeled. The model domain for the half-plume was $80 \mathrm{~m}$ wide. The numerical solution was performed for the resident concentration mADE and flux concentrations were computed from the simulated resident concentrations using Eq. ( 5 b) by numerically evaluating resident concentration gradients.

Flux concentrations versus time are plotted for three lateral distances from the plume centerline ( $y=0,25$ and $55 \mathrm{~m})$ at $x=100 \mathrm{~m}$ and $500 \mathrm{~m}$ (Fig. 6). Analytical and numerical results are in close agreement, except for slight overpredictions at later times and at $y=55 \mathrm{~m}$, which is likely attributable to slight numerical dispersion. Comparison of the 3-D simulated breakthrough curves at $y=0 \mathrm{~m}$ with the $1-\mathrm{D}$ simulations
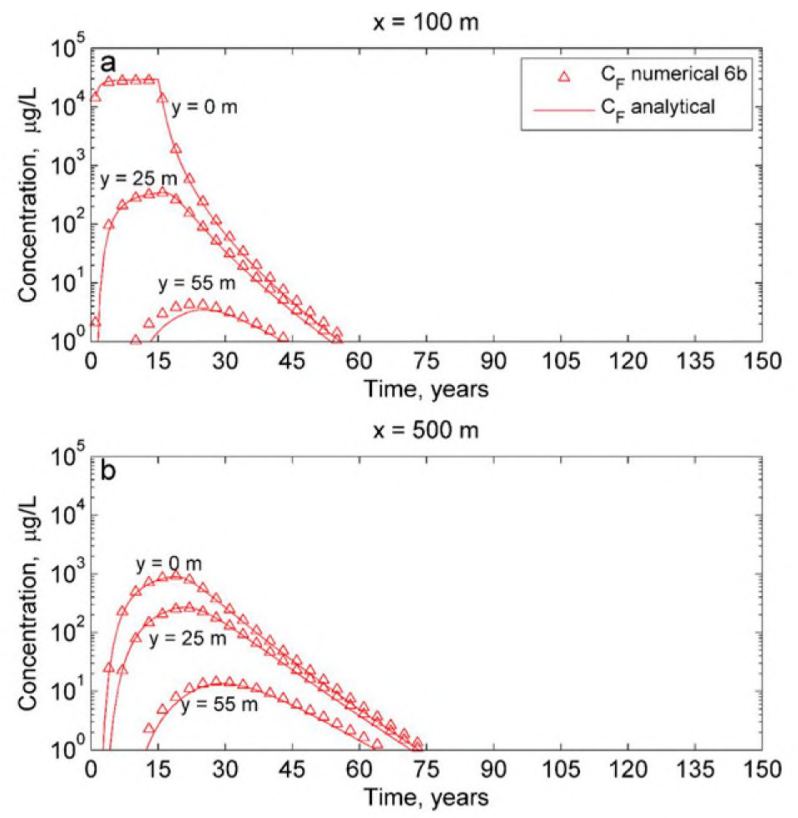

Fig. 6. Analytical and numerical 3-D simulations of flux concentration versus time at $x=100 \mathrm{~m}$ (a) and $500 \mathrm{~m}$ (b) for two lateral distances $(y)$ from the plume centerline.

reveals faster attenuation over time for the $3-\mathrm{D}$ case, which is the anticipated result due to lateral and vertical dispersion.

Resident concentrations versus $x$-axis distance at $t=$ 25 years at three depths ( $z=0,5$ and $10 \mathrm{~m}$ ) show a vertical concentration gradient at $x=0$, which diminishes downgradient (Fig. 7). Close agreement between numerical and analytical solutions indicates that lateral and vertical dispersion terms and boundary reflection terms in the vertical dimension for the analytical solution are correct and accurate.

\subsection{Verification of the upscaled dispersion model}

The upscaling relations given by Eqs. (19a)-(19i) may be derived by equating certain characteristics of the mono- and bicontinuum models, such as their first- and second-moments, which will yield equality of the matched characteristic, but not equality at every point in time or space. In this section, we investigate applications of the upscaled dispersion model to previously published studies of media with significant mass

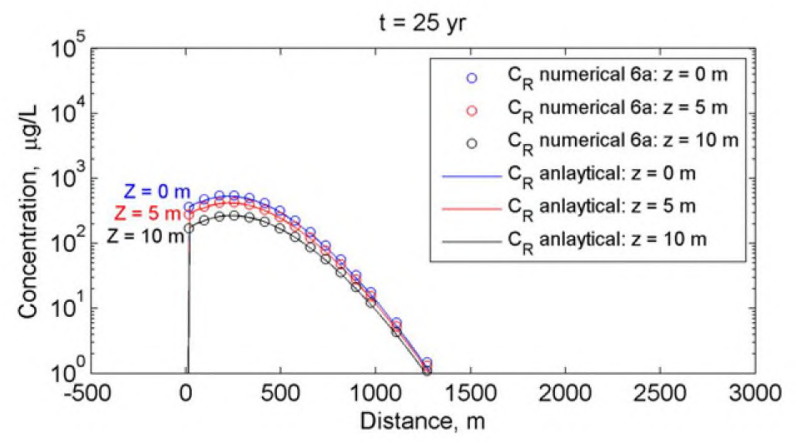

Fig. 7. Analytical and numerical 3-D simulations of resident concentration at $t=25$ years versus distance in $x$-direction for three depths, $z$. 
transfer limitations that exhibit early breakthrough and extended tailing to assess the accuracy of the upscaled dispersion model.

The first problem involves a laboratory study of $190 \mathrm{~mm}$ long columns with an outer diameter of $52 \mathrm{~mm}$ consisting of a sand/clay/grout mixture with a $1.7 \mathrm{~mm}$ diameter hole in the center extending axially from the inlet of the column to the outlet of the column intended to simulate a root or worm channel (Parker, 1984). The porosity was 0.365. A 0.65 pore volume bromide solution was added to two essentially identical columns under positive pressure at a flow rate of $4.65 \times 10^{-4} \mathrm{~m} / \mathrm{s}$. One column was sectioned immediately following tracer injection, cut into $19 \mathrm{~mm}$ long sections and extracted pore fluid was analyzed for bromide. Bromide-free solution was added to the second column for another 1.15 pore volumes and effluent samples were collected and analyzed. No measurement of the effective diffusion coefficient was available. However, Promentilla et al. (2009) report diffusion tortuosities for cement samples over a range of porosities, which in conjunction with a literature value for the bromide diffusion coefficient in bulk water yield an effective diffusion coefficient of $7.4 \times 10^{-9} \mathrm{~m}^{2} / \mathrm{s}$. Disregarding mobile zone dispersivity, an effective dispersivity of $167 \mathrm{~m}$ was computed for the column from Eq. (19a) using the "hollow cylinder" geometry factor.

The experimental data reveal extremely rapid breakthrough and extended "tailing" albeit at very low concentrations due slow back-diffusion from the matrix and high dilution in mobile region (Fig. 8a). Except for the sample closest to the inlet, measured resident concentrations after the injection period and prior to flushing are less than $3 \%$ of the effluent concentration magnitude and the time of sampling (Fig. 8b). The higher concentration in the sample close to the inlet is attributable to longitudinal diffusion from the upper surface of the column
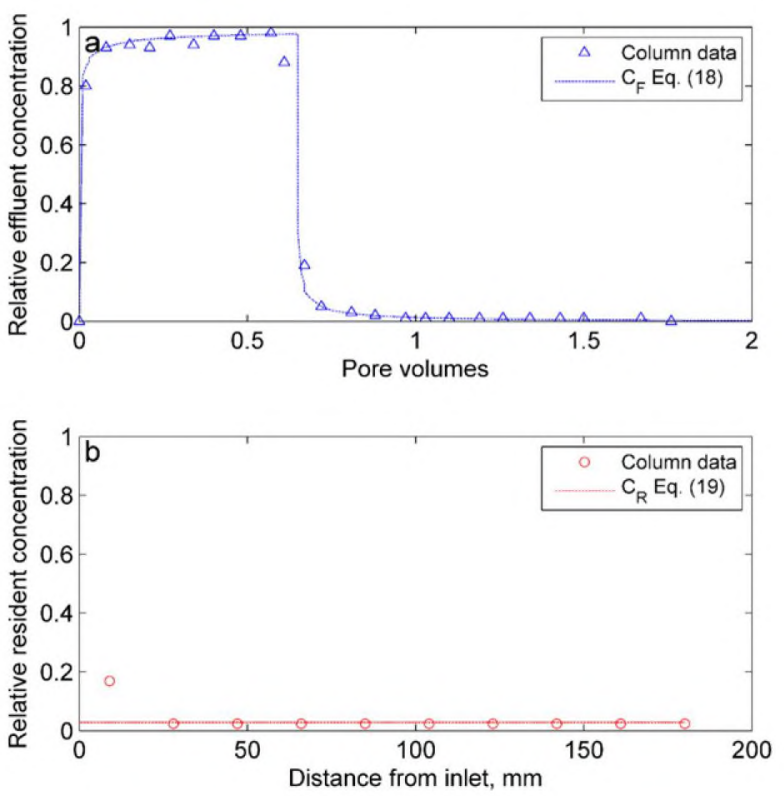

Fig. 8. Observed and simulated transport for Parker (1984) column experiment with "wormhole": (a) measured and simulated breakthrough curves, (b) measured and simulated resident concentrations versus distance immediately following 0.65 pore volume tracer injection. rather than radial diffusion from the "wormhole."

Simulated flux concentrations using model parameters computed using Eqs. (19a)-(19i) without any calibration match measured effluent data and simulated resident concentrations agree well with the sectioned column data (Fig. 8). Note that simulated and measured flux concentrations at the end of the injection period are much greater than measured and simulated resident concentrations. The importance of distinguishing between resident and flux concentrations and of employing the correct governing equation and boundary conditions should be self-evident. This example provides compelling evidence that the mono-continuum dispersion model is capable of describing transport behavior in media characterized by highly preferential flow paths.

The second problem we consider involves a high resolution 2-D numerical simulation by Parker et al. (2008) of chlorinated solvent in a permeable aquifer with discontinuous clay layers underlain by a clay aquitard. The model was used to simulate advection-dominated transport in the permeable material and diffusion in low permeability zones (Fig. 9). Due to the large contrast between advective and diffusive time scales, a very high resolution grid and small time steps were required. Pools of TCE were assumed to occur on clay lenses as constant concentration sources for 30 years, after which sources were completely removed. Note that since mobile zone dispersivity is small, local scale resident and flux concentrations will be essentially identical and high resolution model results will be insensitive to the type of boundary condition employed. A multi-level monitoring well was assumed to be installed above the aquitard near the downgradient boundary about $160 \mathrm{~m}$ from the sources. Numerically simulated TCE concentrations at most depths in the well remained above $1 \mu \mathrm{g} / \mathrm{L}$ following 200 years of flushing with clean water after source removal as a result of back-diffusion from the clay layers (Fig. 9).

Since contaminant sources are distributed more or less uniformly over the aquifer thickness, the 2-D numerical simulation may be reasonably approximated as a verticallyaveraged 1-D problem. If we approximate the model domain

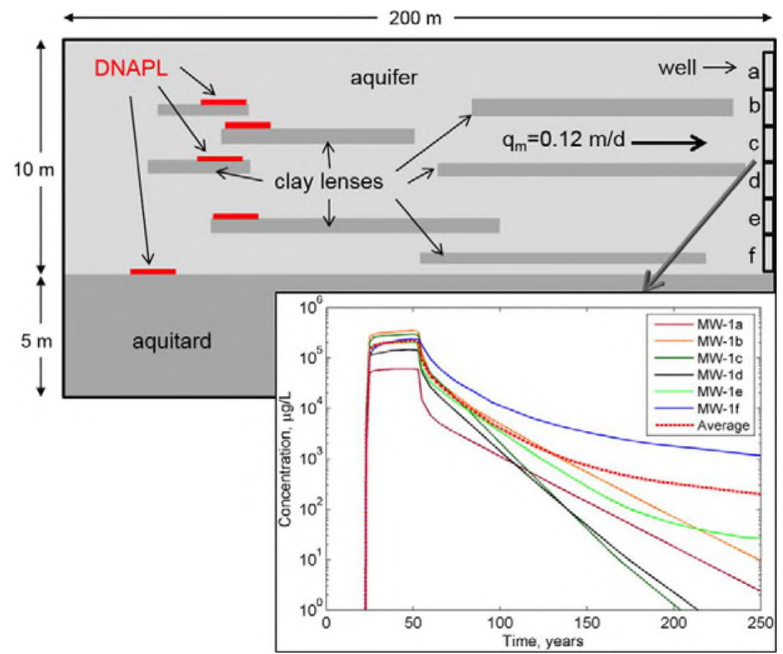

Fig. 9. Domain for high resolution numerical model and numerically simulated TCE concentrations at different depths plus depth-averaged concentration in downgradient well over time (Parker et al., 2008). 
as a system consisting of horizontal mobile and immobile layers, a flux concentration solution of the upscaled dispersion model may be obtained using parameters given by Eqs. (19a)-(19i) to simulate samples taken from the entire well length. The model domain is taken as the $15 \mathrm{~m}$ thickness that includes the aquifer and aquitard. The well is not screened below the top of the aquitard and the well bore does not intersect any clay lenses within the aquifer. Therefore, the arithmetic average of numerically computed concentrations over all well intervals within the $10 \mathrm{~m}$ thick aquifer represent a flow-weighted concentration over the 15 m deep model domain.

Mobile and immobile zone porosities for this problem were assumed to be 0.4 . Retardation factors were 1 in mobile zones, 5 in clay lenses within the aquifer and 3 in the aquitard. The mobile zone Darcy velocity is $0.12 \mathrm{~m} / \mathrm{d}$ and the effective TCE diffusion coefficient in all clay units was assumed to be $3.9 \times 10^{-5} \mathrm{~m}^{2} / \mathrm{d}$. If we consider only effects of mass transfer involving clay lenses within the aquifer, the mobile pore fraction, $f_{m}$, over the $10 \mathrm{~m}$ thick aquifer is 0.85 and the average diffusion path length, $L_{i m}$, is $0.25 \mathrm{~m}$. Plugging the foregoing values into Eqs. (19a)-(19i) gives an effective longitudinal dispersivity of $201 \mathrm{~m}$. The retarded pore velocity (averaged over the full $15 \mathrm{~m}$ depth) is $58 \mathrm{~m} / \mathrm{year}$. Average mADE model flux concentration at the monitoring well simulated with these upscaled model parameters dropped below $1 \mu \mathrm{g} / \mathrm{L}$ about 85 years after source removal (results not shown). Since the high resolution numerical model showed average concentrations exceeding $100 \mu \mathrm{g} / \mathrm{L}$ after 200 years, it is evident that the thin clay layers within the aquifer are relatively minor contributors to back-diffusion processes.

If we include the $5 \mathrm{~m}$ thick aquitard in the tally of immobile zones, the mobile pore fraction over the $15 \mathrm{~m}$ model thickness drops to 0.57 and the volume-weighted diffusion path length increases to $3.9 \mathrm{~m}$. Note that the diffusion path length for the aquitard is equal to its thickness, not its half thickness, because it contacts the aquifer on only one side. Although the aquitard retardation factor is smaller than that for the thin clay lenses, the average aquitard is about 5 times more voluminous resulting in a lower average pore velocity of $31 \mathrm{~m} /$ year. Eq. (19a) gives a longitudinal dispersivity of $27,000 \mathrm{~m}$ for this system. With this exceedingly high dispersivity, the upscaled mADE model predicts earlier breakthrough than the high resolution model and exhibits a faster drop in concentration following source removal compared to the high resolution numerical model (Fig. 10). However, the late-time rate of concentration change for the upscaled model closely parallels the numerical model.

The early time deviations in the upscaled model likely reflect shorter effective diffusion path lengths at times when contaminant has only penetrated a fraction of the low permeability zones. To account for this, we investigated the simple and widely-used approximation of diffusion path length timedependence (Crank, 1975)

$L_{i m}(t) \approx \min \left(L_{i m}^{\max },\left(\frac{2 D_{i m} t}{R_{i m}}\right)^{1 / 2}\right)$

where $L_{m m}^{\max }$ is the maximum diffusion path length $(5 \mathrm{~m}), t$ is time since the initial release, and other variables are as previously defined. Substitution of Eq. (21) into Eq. (19a) yields a linear increase in dispersivity with time to a maximum value of $6800 \mathrm{~m}$ in 230 years. Simulated flux concentrations using

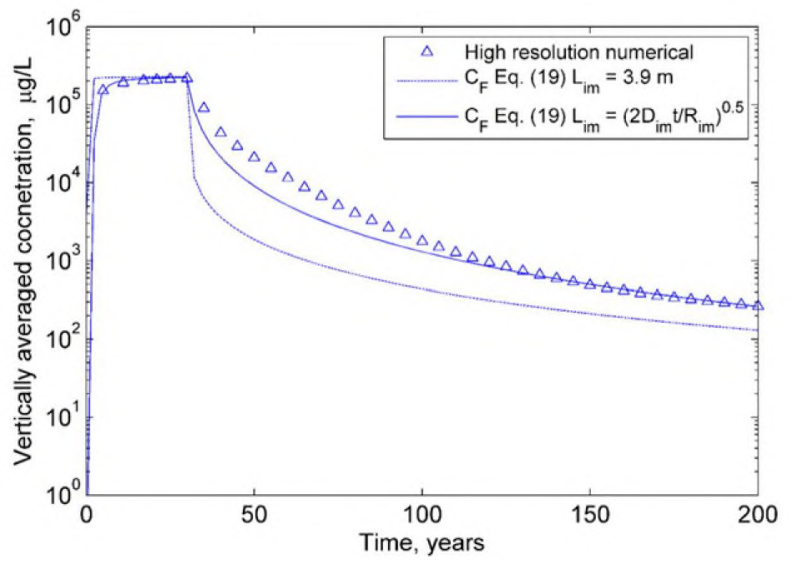

Fig. 10. Vertically-averaged flux concentrations in aquifer with clay layers at well location based on Parker et al. (2008) high resolution numerical simulation and results for upscaled dispersion model with a constant diffusion path length. $L_{\mathrm{jm}}$ of $3.9 \mathrm{~m}$, or with a time-varying $L_{\mathrm{im}}(t)$.

the time-dependent diffusion length and dispersivity in the upscaled model follow the high resolution numerical results fairly well except for moderate under-prediction between 30 and 100 years (Fig. 10). The maximum error during this period is about $65 \%$, which would likely be within the range of "noise" in field data and the limits of reliability that can be reasonably expected even with sophisticated models at such a complex site. In any case, the upscaled model appears to offer a practical and efficient approach to evaluating the effects of back-diffusion that arise at heterogeneous sites, especially considering the demonstrated feasibility of quantifying dispersivity from physically meaningful site parameters with little or no calibration.

Comparisons between upscaled model $C_{R}$ solutions and numerical model results were not possible since simulated aquitard concentrations were not given by Parker et al. (2008). However, a few observations may be made. The analytical model indicates that resident concentrations are less than flux concentrations prior to source removal and greater after source removal. Further, the magnitude of the difference between $C_{R}$ and $C_{F}$ may be shown to increase with dispersivity. These observations are readily explained in the context of the upscaled mobile-immobile model. When the source is active, concentrations are higher in mobile zones than adjacent immobile zones, resulting in higher volume-averaged than flow-averaged concentrations, while the reverse is true after source removal. Also, the magnitude of concentration differences between mobile and immobile zones will increase as diffusive mass transfer resistance increases, which corresponds to increasing dispersivity according to Eq. (19a).

As a final point of interest, consider the fact that the well in the numerical model is located within a "window" in the aquifer where no clay layers intersect the well bore over the $10 \mathrm{~m}$ aquifer thickness. As a result, volume- and flow-averaged concentrations over the $10 \mathrm{~m}$ aquifer thickness (i.e., excluding the aquitard) are essentially equal at this location. In spite of the absence of clay lenses at the monitoring location, the flux concentration solution for the upscaled model with timedependent dispersion provides a reasonable approximation of the high resolution numerical results. This reflects the relative insensitivity of flux concentrations to local variations in the 
immobile fraction, since the later have zero contribution to flux concentration. As discussed previously, flux concentrations are controlled by cumulative upgradient transport processes (Fig. 2). In contrast, local resident concentrations will exhibit large variance in response to local spatial variability in the distribution of low permeability material. For these reasons, well measurements, if properly interpreted as flux concentrations, are expected to exhibit less variance and hence be more useful for model calibration than resident or soil concentration data.

\section{Summary and conclusions}

Hydrodynamic dispersion is fundamentally a random process driven by spatial and in some cases temporal variations in fluid velocity. Consideration of constraints on hydrodynamic dispersion upgradient of source locations and distinctions between resident and flux concentrations is critical for the successful application of advection-dispersion models to heterogeneous systems. Unlike molecular diffusion, which is independent of fluid velocity, hydrodynamic dispersion cannot occur counter to the mean flow direction. Because the intrinsic space scale of hydrodynamic dispersion is much greater than that of diffusion, variations in fluid velocity at larger scales introduce potential sampling and measurement difficulties. Depending on how samples are taken, fluid may be obtained equally from all pores (volume-averaged resident concentrations) or in proportion to local permeability (flow-averaged flux concentrations). Water samples from wells or suction sampling devices should generally be regarded as flux concentrations, while soil samples that are mixed and extracted in their entirety may be regarded as resident concentrations after correcting for dilution and sorption. The scale of measurements versus that of significant heterogeneities must be carefully considered for upscaled models in order to properly interpret field data, e.g., for selection of Eqs. (20a)-(20e).

Although the mADE is normally derived and written in terms resident concentrations, a flux concentration MADE may be derived that has the same mathematical form as that for resident concentration. However, solution of the flux concentration MADE requires a transformation of boundary conditions to maintain consistency with resident concentration mADE solutions. In particular, while the resident concentration mADE requires specification of a contaminant source in terms of its flux, solution of the flux concentration mADE requires specification of the boundary condition as a flux concentration. If an existing plume is modeled by specifying an initial contaminant distribution based on field data, flux concentrations must be specified for the flux mADE and resident concentrations for the resident mADE. Care must be exercised to ensure consistency between the desired solution type and the type of data. An interesting and less appreciated property of flux concentrations is that they can be viewed as rates of change in the space integral of all upgradient resident concentrations. As a consequence, flux concentrations are less sensitive than resident concentrations to local variations in aquifer properties near the sampling location. This characteristic makes well samples much more useful, in general, for model calibration and verification than resident or soil samples.

Upstream dispersion can be avoided in analytical solutions by placing the source at the origin of a semi-infinite domain in the direction of groundwater flow. If multiple sources need to be modeled, solutions for each source can be simulated in local coordinates and superposed after translating to a regional coordinate system. The same strategy may be adopted for numerical solutions for single sources, but for multiple sources the most robust strategy is to solve the resident concentration formulation of the mADE and specify zero or low hydrodynamic dispersion for grid cells immediately upgradient of each source to prevent non-physical upstream dispersion (upstream diffusion may be permitted). Flux concentrations may be computed from resident concentration simulations by Eqs. (5a) and (5b), which requires numerical evaluation of resident concentration gradients.

Errors in specification of downstream boundaries can also adversely affect numerical solution accuracy. A zero concentration gradient at or near the downgradient edge of the plume should be specified. If the entire plume is not required to be modeled, a zero gradient boundary may be imposed at a distance $x$ from the nearest source without greatly affecting the upstream solution if $x$ is much greater than the longitudinal dispersivity. Otherwise, the boundary condition will force a large dispersive flux at the boundary to be zero, which will induce errors upstream. If the plume intercepts a seepage boundary (e.g., soil column exit or stream bed) and downgradient transport processes are not explicitly modeled, it may be necessary to extend the model domain past the seepage face to a similar distance from the nearest source to prevent avoid a zero gradient condition from propagating solution errors upgradient.

Heterogeneous aquifers frequently exhibit much earlier breakthrough downgradient of a source than expected for purely advective transport and show extended tailing to long travel times. It is commonly assumed that simple mADE models are not capable of describing such systems. We have shown this to be a misconception. While the MADE may not describe every detail of such systems exactly, when distinctions between resident and flux concentration solutions, dispersion upgradient of sources, and time-dependence of diffusion path length are addressed, the mADE is capable of representing rapid breakthrough and extended tailing with reasonable accuracy even in quite extreme cases.

Furthermore, we have shown that expressions for apparent dispersivity and other parameters in the MADE model may be explicitly formulated in terms of physically-defined variables in models for diffusion-limited mobile-immobile transport. The procedures used to derive these upscaled models are approximate and will generally yield agreement with more rigorous models in terms of lower order (e.g., first and second) moments in time with deviations to be expected in higher order terms. It is interesting to note that Parker and Valocchi (1986) reported deviations between rigorous diffusion-limited mobile-immobile simulations and upscaled ADE solutions were smaller than those between diffusion-limited models and firstorder mass transfer approximations, which are commonly regarded as a more rigorous means of dealing with mobileimmobile type systems than simpler mADE models.

While this paper has focused on the interpretation of apparent dispersivity in the context of physical nonequilibrium, kinetically-controlled chemical reactions can result in similar behavior. Toride et al. (1999) discuss the mathematical equivalence of first-order mobile-immobile models and two-site chemical nonequilibrium models, while Parker and Valocchi 
(1986) and van Genuchten and Dalton (1986) have derived upscaled dispersivities in terms of first-order mobile-immobile model parameters. Therefore, upscaling relations could easily be formulated in terms of chemical kinetic model parameters, which would yield early breakthrough and extended tailing characterisitic analogous to those demonstrated in the present paper.

The upscaled mADE model was able to accurately simulate measured resident and flux concentrations for a laboratory column experiment characterized by extreme early breakthrough and extended tailing without any parameter calibration. The mADE model was also able to provide a reasonable approximation of high resolution numerical simulations of an aquifer with multiple clay lenses and an aquitard that exhibited prolonged back-diffusion. Upscaled model simulations using a constant aquitard diffusion path length overpredicted the initial rate of concentration decrease following source removal. This deficiency was largely eliminated by using a time-dependent diffusion path length in the upscaling equations. Again, no calibration was performed to fit any model parameters.

Most 2-D and 3-D analytical transport models, including widely used solutions by Domenico (1987); Galya (1987), and Wexler (1992), do not distinguish resident and flux concentrations and allow upgradient dispersion. Exceptions include Batu and van Genuchten (1990) and Leij et al. (1991) models which have broad applicability but are rather computationally intensive. The analytical model described here explicitly distinguishes between resident and flux concentrations, precludes back-dispersion, and is computationally efficient. We have shown here that the model is capable of describing both resident and flux concentrations for problems that would commonly be regarded as intractable for a mADE model. Analytical model results agree closely with numerical simulations using MT3D when appropriate care is taken to follow guidelines for application of numerical models to heterogeneous sites when much or all of the heterogeneity is modeled implicitly (i.e., using "effective" parameters) rather than explicitly.

Our objective in developing the analytical model was to obtain a method to simulate contaminant transport at complex sites in response to natural and engineered processes for use in a stochastic cost-optimization protocol for remediation design and site management. Since thousands of simulations are required for site analyses that explicitly consider model and measurement uncertainty, a small sacrifice in model rigor in exchange for a high degree of efficiency and robustness was considered a favorable tradeoff.

It is our conviction that the mADE, or any other potential modeling approach, should not be accepted or rejected out of hand as suitable or not suitable for a given application without careful consideration of its advantages and limitations. Monocontinuum ADE models will not provide perfect representations of diffusion-limited transport, but they can provide a reasonable approximation that may be "good enough" when sources of prediction uncertainty are taken into consideration. The same can be said of all models which - no matter how sophisticated - are still merely approximations of reality. The "best" model will always be a tradeoff between potential accuracy, efficiency, robustness, and cost, balanced against project objectives, available data, budget and other factors.

\section{Acknowledgment}

This research was conducted with funding from the U.S. Department of Defense Strategic Environmental Research and Development Program (SERDP) Environmental Restoration Program managed by Dr. Andrea Leeson under project ER2310 entitled "A practical approach for remediation performance assessment and optimization at DNAPL sites for early identification and correction of problems." The first author is grateful for many discussions with Rien van Genuchten on issues addressed in this paper over the past 30 years.

\section{References}

Ball, W.P., Liu, C., Xia, G., Young, D.F., 1997. A diffusion-based interpretation of tetrachloroethene and trichloroethene concentration profiles in a groundwater aquitard. Water Resour. Res. 33, 2741-2757.

Barker, J.A., 1985. Block-geometry functions characterizing transport in densely fissured media. J. Hydrol. 77, 263-279.

Barry, D.A. Parker, J.C., 1987. Approximations for solute transport through porous media with flow transverse to layering. Transp. Porous Media 2 , $65-82$.

Batu, V., 1996. A generalized three-dimensional analytical solute transport model for multiple rectangular first-type sources. ]. Hydrol. 174, 57-82.

Batu, V., van Genuchten, M.T., 1990. First- and third-type boundary conditions in two-dimensional solute transport modeling. Water Resour. Res. 26, 339-350.

Berkowitz, B., Cortis, A., Dentz, M., Scher, H., 2006. Modeling non-Fickian transport in geological formations as a continuous time random walk. Rev. Geophys. 44. http://dx.doi.org/10.1029/2005RG000178.

Bolt, G.H., 1979. Movement of solutes in soil: principles of adsorption/exchange chromatography. In: Bolt, G.H. (Ed.), Developments in Soil Science 5BSoil Chemistry, B. Physico-chemical Models. Elsevier, Amsterdam, pp. 295-348

Bromly, M., Hinz, C., 2004. Non-Fickian transport in homogeneous unsaturated repacked sand. Water Resour. Res. W07402. http:/dx.doi.org/10.1029/ 2003 WR002579.

Chapman, S.W., Parker, B.L, 2005. Plume persistence due to aquitard back diffusion following dense nonaqueous phase liquid source removal or isolation. Water Resour. Res. 41, W12411. http://dx.doi.org/10.1029/2005 WR004224.

Cleary, R.W., Ungs, M.]., 1978. Analytical methods for groundwater pollution and hydrology. Water Resour. Prog. Rep. 78-WR-15. Princeton University, Princeton, New Jersey.

Coats, K.H., Smith, B.D., 1964. Dead-end pore volume and dispersion in porous media. Soc. Petrol. Eng. J. 4, 73-84

Crank, J., 1975. The Mathematics of Diffusion. 2nd ed. Oxford University Press London (414 pp.).

De Smedt, F., Wierenga, P.J., 1979. A generalized solution for solute flow in soils with mobile and immobile water. Water Resour. Res. 15, 1137-1141.

Dentz, M., Le Borgne, T., Englert, A., Bijeljic, B., 2011. Mixing, spreading and reaction in heterogeneous media: a brief review. J. Contam. Hydrol. $120-121,1-17$

Domenico, P.A., 1987. An analytical model for multidimensional transport of a decaying contaminant species. J. Hydrol. 91, 49-58.

Falta, R. Basu, N., Rao, R., 2005. Assessing impacts of partial mass depletion in DNAPL source zones: II. Coupling source strength functions to plume evolution. J. Contam. Hydrol. 79, 45-66.

Galya, D.P., 1987. A horizontal plane source model for ground-water transport. Ground Water 25, 733-739.

Gelhar, L.W., Welty, C., Rehfeldt, K.R., 1992. A critical review of data on field scale dispersion in aquifers. Water Resour. Res. 28, 1955-1974.

van Genuchten, M.T., 1985. A general approach to modeling solute transport in structured soils. Proc. 17th Int. Congress on Hydrogeology of Rocks of Low Permeability, Memoires Int. Assoc. of Hydrogeologists. 17, pp. 513-526.

van Genuchten, M.T., Dalton, F.N., 1986. Models for simulating salt movement in aggregated field soils. Geoderma 30, 165-183.

van Genuchten, M.T., Parker, J.C., 1984. Boundary conditions for displacement experiments through short laboratory soil columns. Soil Sci. Soc. Am. 48, 703-708.

Gillham, R.W., Sudicky, E.A. Cherry, JA., Frind, E.O., 1984. An advectiondiffusion concept for solute transport in heterogeneous unconsolidated geological deposits. Water Resour. Res. 20, 369-378.

Guyonnet, D., Neville, C., 2004. Dimensionless analysis of two analytical solutions for 3-D solute transport in groundwater. ]. Contam. Hydrol. 75 141-153. 
Huyakorn, P., Ungs, M., Mulkey, L., Sudicky, E., 1987. A three-dimensional analytical method for predicting leachate migration. Ground Water 25, $588-598$.

Kreft, A., Zuber, A., 1978. On the physical meaning of the dispersion equation and its solution for different initial and boundary conditions. Chem Eng. Sci. 33, 1471-1480.

Leij, F.]., Dane, J.H., 1990. Analytical solution of one-dimensional advection equation and two- and three-dimensional dispersion equation. Water Resour. Res. 26, 1475-1482.

Leij, F., Skaggs, T., van Genuchten, M.T., 1991. Analytical solutions for solute transport in three-dimensional semi-infinite porous media. Water Resour. Res. 27, 2719-2733.

Levy, M., Berkowitz, B., 2003. Measurement and analysis of non-Fickian dispersion in heterogeneous porous media. J. Contam. Hydrol. 64, 203-226.

Liu, C., Ball, W.P., 2002. Back diffusion of chlorinated solvent contaminants from a natural aquitard to a remediated aquifer under well-controlled field conditions: predictions and measurements. Ground Water 40, 175-184.

Liu, C., Ball, W.P., Ellis, J.H., 1998. An analytical solution to one-dimensional solute advection-dispersion equation in multi-layer porous media. Transp. Porous Media 30, 25-43.

Liu, C., Szecsody, J.E., Zachara, J.M., Ball, W.P., 2000. Use of the generalized integral transform method for solving equations of solute transport in porous media Adv. Water Resour. 23, 483-492

Neuman, S.P., Tartakovsky, D.M., 2009. Perspective on theories of non-Fickian transport in heterogeneous media. Adv. Water Resour. 32, 670-680.

Ogata, A., Banks, R. 1961. A solution of the differential equation of longitudinal dispersion in porous media. Prof. Pap. No. 411A. USGS, Reston, Virginia.

Parker, J.C., 1984. Analysis of solute transport in column tracer studies. Soil Sci. Soc. Am. J. 48, 719-724.

Parker, J.C., Valocchi, A.J., 1986. Constraints on the validity of equilibrium and first-order kinetic transport models in structured soils. Water Resour. Res. $22,399-407$.

Parker, J.C., van Genuchten, M.T., 1984. Flux-averaged and volume-averaged concentrations in continuum approaches to solute transport. Water Resour. Res. 20, 866-872.

Parker, B.L., Chapman, S.W., Guilbeault, M.A., 2008. Plume persistence caused by back diffusion from thin clay layers in a sand aquifer following TCE sourcezone hydraulic isolation. J. Contam. Hydrol. 102, 86-104.

Parker, J.C., Kim, U., Kitanidis, P., Cardiff, M., Liu, X, Lee, J., 2011. Practical costoptimization of characterization and remediation decisions at DNAPL sites with consideration of prediction uncertainty. SERDP Project ER-1611, Fina! Report, May 2011.
Passioura, J.B., 1971. Hydrodynamic dispersion in porous media. l. Theory. Soil Sci. 111, 339-344.

Promentilla, M.A., Sugiyama, T., Hitomi, T., Takeda, N., 2009. Quantification of tortuosity in hardened cement pastes using synchrotron-based X-ray computed microtomography. Cem. Concr. Res. 39, 548-557.

Raats, P.A.C., 1981. Transport in structured porous media. Proc. Euromech. 143, Sept 2-4, Delft, pp. 221-226.

Rasmuson, A. Neretnieks, I., 1980. Exact solution for diffusion in particles and longitudinal dispersion in packed beds. Am. Inst. Chem. Eng.J. 26, 686-690.

Roth, K., Jury, W.A., 1993. Linear transport models for adsorbing solutes. Water Resour. Res. 29, 1195-1203.

Rubin, S., Dror, l., Berkowitz, B., 2012. Experimental and modeling analysis of coupled non-Fickian transport and sorption in natural soils. J. Contam Hydrol. 132, 28-36.

Sagar, B., 1982. Dispersion in three dimensions: approximate analytical solutions. J. Hydraul. Div. ASCE 108, 47-62.

Seyedabbasi, M.A.C.J., Newell, D.T.A., Sale, T.C., 2012. Relative contribution of DNAPL dissolution and matrix diffusion to the long-term persistence of chlorinated solvent source zones. J. Contam. Hydrol. 134-135, 69-81. http://dx.doi.org/10.1016/j.jconhyd.2012.03.010.

Starr, R.C., Gillham, R.W., Sudicky, E.A, 1985. Experimental investigation of solute transport in stratified porous media 2 . The reactive case. Water Resour. Res. 21, 1043-1050.

Sudicky, E.A., Gillham, R.W., Frind, E.O., 1985. Experimental investigation of solute transport in stratified porous media 1 . The nonreactive case. Water Resour. Res. 21, 1035-1041.

Tang, D.H., Frind, E.O., Sudicky, E.A., 1981. Contaminant transport in fractured porous media: analytical solution for a single fracture. Water Resour. Res. $17,555-564$

Toride, N., Leij, FJ. van Genuchten, MT., 1999. CXTFT code for estimating transport parameters from laboratory or field tracer experiments. Version 2.1. U.S. Salinity Laboratory Research Report No. 137, USDA, ARS, Riverside, CA.

Wexler, E.]., 1992. Analytical solutions for one- two- and three-dimensional solute transport in ground-water systems with uniform flow. U.S Geological Survey Techniques of. Water-Resources Investigations, Book 3 , Chap. Series 03-B7B7. U.S. Geological Survey $190 \mathrm{pp}$.

Yeh, G.T., Tsai, Y.-J., 1976. Analytical three-dimensional transient modeling of effluent discharges. Water Resour. Res. 12, 533-540.

Zhang, Y., Baeumer, B., Benson, D.A., 2006. Relationship between flux and resident concentrations for anomalous dispersion. Geophys. Res. Lett. 33 L18407. http://dx.doi.org/10.1029/2006GL027251. 